\title{
Remediation of $\mathrm{Cr}(\mathrm{VI})$ by biogenic magnetic nanoparticles: An x-ray magnetic circular dichroism study
}

\author{
N. D. Telling ${ }^{1}$, V. S. Coker ${ }^{1}$, R. S. Cutting ${ }^{1}$, G. van der Laan ${ }^{1,2}$, C. I. Pearce ${ }^{1}$, R. A. D. \\ Pattrick $^{1}$, E. Arenholz ${ }^{3}$, and J. R. Lloyd ${ }^{1}$ \\ ${ }^{1}$ School of Earth, Atmospheric and Environmental Sciences, University of \\ Manchester, Manchester M13 9PL, UK \\ ${ }^{2}$ Magnetic Spectroscopy Group, Diamond Light Source, Didcot, Oxfordshire, OX11 \\ $O D E, U K$ \\ ${ }^{3}$ Advanced Light Source, Lawrence Berkeley National Laboratory, Berkeley, CA, USA
}

Biologically synthesized magnetite $\left(\mathrm{Fe}_{3} \mathrm{O}_{4}\right)$ nanoparticles are studied using x-ray absorption and $\mathrm{x}$-ray magnetic circular dichroism following exposure to hexavalent $\mathrm{Cr}$ solution. By examining their magnetic state, $\mathrm{Cr}$ cations are shown to exist in trivalent form on octahedral sites within the magnetite spinel surface. The possibility of reducing toxic $\mathrm{Cr}(\mathrm{VI})$ into a stable, non-toxic form, such as a $\mathrm{Cr}^{3+}$-spinel layer, makes biogenic magnetite nanoparticles an attractive candidate for $\mathrm{Cr}$ remediation.

PACS number(s): 91.25.fa; 87.64.kd; 89.60.-k; 91.62.Rt 
Magnetic nanoparticles have potential applications in areas as diverse as cancer therapies $^{1}$ and magnetoelectronic devices ${ }^{2,3}$. Their enhanced surface catalytic properties also make them very suitable for oxidation-reduction (redox) reactions ${ }^{3}$. One such reaction that has important environmental and health consequences involves the reduction and immobilization of highly toxic $\mathrm{Cr}^{6+}$ cations, found in $\mathrm{Cr}$ contaminated groundwaters, to their less harmful $\mathrm{Cr}^{3+}$ counterpart ${ }^{4,5}$. In this Letter we describe the use of x-ray absorption (XA) and x-ray magnetic circular dichroism (XMCD) to probe the near surface region of biologically synthesized (biogenic) magnetite nanoparticles, following exposure to varying concentrations of $\mathrm{Cr}^{6+}$ containing solution. Biogenic magnetite offers a route to the scaled-up nanoparticle production required for $\mathrm{Cr}$ remediation, whilst using environmentally friendly preparation methods. Previous $\mathrm{Cr}$ remediation studies using synthetic zero-valent $\mathrm{Fe}$ and iron oxide surfaces suggested the formation of $\mathrm{Cr}^{3+}$-rich oxide and oxy-hydroxide surface layers ${ }^{4-6}$. In contrast, the results presented here indicate the incorporation of $\mathrm{Cr}^{3+}$ cations into octahedral sites of the magnetite spinel structure.

Highly crystalline biogenic magnetite nanoparticles with average dimensions of $\sim 20 \mathrm{~nm}$ were obtained from a ferrihydrite suspension starting material using the bacterium Geobacter sulfurreducens, as described in detail elsewhere ${ }^{7,8}$. The nanoparticles were subsequently exposed to a solution of $\mathrm{Cr}(\mathrm{VI})$ (potassium chromate) at varying concentrations of $0.5 \mathrm{mM}, 1 \mathrm{mM}$, and $5 \mathrm{mM}^{8}$. Batch chromate reduction experiments clearly showed that the biogenic magnetite nanoparticles were successful at reducing $\mathrm{Cr}(\mathrm{VI})$ for all concentrations used ${ }^{8}$. XA and XMCD measurements were performed on untreated and $\mathrm{Cr}$-exposed samples, using beamline 4.0.2 at the Advanced Light Source, Lawrence Berkeley National Laboratory. Spectra were obtained using total-electron-yield detection, which gives a probing depth of $\sim 4$ $\mathrm{nm}$. Care was taken to ensure that the nanoparticles were maintained in anaerobic conditions at all stages of the experiment by placing them in airtight containers for transport and performing sample handling only in anaerobic environments.

The XA spectra measured across the $\mathrm{Cr}$ and $\mathrm{Fe} L_{2,3}$ edges for the $\mathrm{Cr}$ treated nanoparticles are shown in Fig. 1. From the relative heights of the main $\mathrm{Cr}$ and $\mathrm{Fe} L_{3}$ absorption peaks, the deduced $\mathrm{Cr}$ content in the nanoparticles displays a scaling behaviour with increasing $\mathrm{Cr}(\mathrm{VI})$ concentration, consistent with previous studies on magnetite surfaces reacted with $\mathrm{Cr}$ solution ${ }^{6}$. In addition, a shoulder feature at $\sim 708$ $\mathrm{eV}$ on the $\mathrm{Fe}$ main absorption feature develops with increasing $\mathrm{Cr}$ content (Fig. 1) 
indicating the surface oxidation of magnetite $\mathrm{Fe}^{2+}$ cations to $\mathrm{Fe}^{3+9}$, which is an expected consequence of the redox reaction with $\mathrm{Cr}(\mathrm{VI})$ solution. The same $\mathrm{Fe}^{3+}$ enrichment would also be seen by substituting $\mathrm{Fe}^{2+}$ cations by $\mathrm{Cr}$.

The Cr XA spectrum for the sample treated with $5 \mathrm{mM} \mathrm{Cr}(\mathrm{VI})$ solutions is compared to standard compounds in Fig. 2(a). Samples treated with lower Cr(VI) concentrations displayed almost identical spectra. It can be seen that the nanoparticle spectrum is very similar to that obtained from the trivalent $\mathrm{Cr}$ compound and varies greatly from the $\mathrm{Cr}^{6+}$ containing compound. There is no obvious contribution from $\mathrm{Cr}^{6+}$ to the XA spectrum, suggesting that the surface contains predominately $\mathrm{Cr}^{3+}$ cations. Furthermore, the XA spectral shape indicates an octahedral site symmetry for the $\mathrm{Cr}$ cations ${ }^{6,10,11}$, in agreement with the strong site preference due to the favourable fit of the charge distribution of the $\mathrm{Cr}^{3+}$ ions in the crystal field at an octahedral site.

A more quantitative analysis can be performed using XMCD, i.e. the difference of XA spectra measured using circularly polarized $\mathrm{x}$-rays in a $0.6 \mathrm{~T}$ magnetic field applied parallel and antiparallel to the X-ray beam direction. The XMCD spectra for the Cr-treated nanoparticles are shown in Fig. 2(b). A clear dichroism is observed for all the samples, indicating the presence of magnetic $\mathrm{Cr}$ cations in the nanoparticle surface region. The sign of the Cr XMCD signal compared to that of the Fe confirms the octahedral site occupation. The XMCD spectra shown in Fig. 2(b) are almost identical to those measured from $\mathrm{CrFe}_{2} \mathrm{O}_{4}$ spinel ferrite powders ${ }^{11}$, and are well reproduced by a multiplet calculation using a charge-transfer model with trivalent $\mathrm{Cr}$ cations on octahedral sites (Fig. 2(b)). The close similarity of the XMCD obtained here with that presented in Ref. 11 would thus imply that at least some of the Cr must be incorporated into the magnetite spinel structure. However, it can also be seen from Fig. 2(b) that the relative strength of the XMCD signal is weaker for higher $\mathrm{Cr}$ content samples. Thus only a portion of the $\mathrm{Cr}$ is able to incorporate into the spinel structure and so to become magnetically polarized. Additonal $\mathrm{Cr}$ must reside in nonmagnetic (or antiferromagnetic) $\mathrm{Cr}^{3+}$-rich surface phases, in agreement with previous observations $^{4-6}$

The Fe cation site occupation in the nanoparticles was investigated by analyzing the Fe $L_{2,3}$ XMCD. Spectra obtained over the $L_{3}$ absorption region are shown for as-prepared and Cr-treated samples in Fig. 3(a). The XMCD is the sum of the contributions from the possible cation $\operatorname{sites}^{7,12}$ where the most intense negative and positive peaks correspond to the $\mathrm{Fe}^{2+}$ and $\mathrm{Fe}^{3+}$ octahedral $(\mathrm{Oh})$ and $\mathrm{Fe}^{3+}$ tetrahedral 
(Td) cation sites, respectively (see Fig. 3(a) for a peak assignment). It appears that the effect of $\mathrm{Cr}$ treatment is to remove $\mathrm{Fe}^{2+}$ cations in $\mathrm{Oh}$ sites, as indicated by the reduction in the first negative peak. However a comparison with the XMCD obtained for Co-doped magnetite nanoparticles in which only removal of $\mathrm{Fe}^{2+}$ cations occurs (Fig. 3(b)), ${ }^{7}$ reveals an additional enhancement of the positive peak. This is due to the spectral overlap of the components in the XMCD spectrum such that a decrease in $\mathrm{Fe}^{2+}$ Oh leads to a more pronounced $\mathrm{Fe}^{3+}$ Td peak.

The Fe $L_{3}$ XMCD spectra were fitted using the weighted sum of the theoretically calculated spectra for each Fe site as shown in Fig. 4. As there is no evidence of changes in the tetrahedral site occupation following reaction with $\mathrm{Cr}$ in either this experiment or previous work, the $\mathrm{Fe}^{3+} \mathrm{Td}$ component was fixed in the fitting procedure. From the fitted components shown in Fig. 4(b,c) for the XMCD it can be seen that the reaction with $\mathrm{Cr}$ solution decreases the $\mathrm{Fe}^{2+}$ site occupancy, and increases $\mathrm{Fe}^{3+}$ occupancy, but by unequal amounts. Thus it appears that both $\mathrm{Cr}^{3+}$ incorporation on $\mathrm{Fe}^{2+}$ sites and cation oxidation from $\mathrm{Fe}^{2+}$ to $\mathrm{Fe}^{3+}$ has occurred. The change in $\mathrm{Fe}^{2+}$ and $\mathrm{Fe}^{3+}$ occupancy as a function of $\mathrm{Cr}(\mathrm{VI})$ concentration for the entire sample series is shown in Fig. 5(a). Their net difference in Fig. 5(b) gives an indication of the Cr occupancy. It can be seen from Fig. 5(b) that $\mathrm{Cr}$ is incorporated into the spinel structure even at low initial $\mathrm{Cr}(\mathrm{VI})$ concentrations, as was also found from the $\mathrm{Cr} L_{2,3}$ XMCD spectra. However, significant oxidation of the magnetite was only observed for the sample treated with $5 \mathrm{mM}$ concentration of $\mathrm{Cr}(\mathrm{VI})$ solution.

In summary, we have used XA and XMCD at the Fe and $\mathrm{Cr} L_{2,3}$ edges to probe biogenic magnetite nanoparticles following redox reactions with $\mathrm{Cr}$ (VI) solution. The results show the presence of magnetically polarized $\mathrm{Cr}^{3+}$ cations and indicate the incorporation of $\mathrm{Cr}$ into octahedral sites in the spinel near surface region. Higher $\mathrm{Cr}$ concentrations lead to the additional formation of $\mathrm{Cr}$-rich surface layers, as found in previous studies ${ }^{4-6}$. Analysis of the Fe XMCD spectra enabled us to distinguish between $\mathrm{Fe}^{2+}$ cations replaced due to $\mathrm{Cr}$ substitution, and those oxidized to $\mathrm{Fe}^{3+}$. The ability of the nanoparticles to fully incorporate $\mathrm{Cr}$ into a stable spinel structure at low $\mathrm{Cr}(\mathrm{VI})$ concentrations has huge implications for environmental applications such as ground water remediation, where the hexavalent $\mathrm{Cr}$ concentration is likely to be even lower than that used in this study. 
This work was supported by grants EP/D057310/1 and EP/D058767/1 from EPSRC and BB/E003788/1 from BBSRC. We thank R. L. Kimber for sample preparation. Supported by the U.S. Department of Energy under Contract No. DEAC02-05CH11231. 
1. G. F. Goya, V. Grazú, and M. R. Ibarra, Curr. Nanoscience 4, 1 (2008).

2. W. Wang, M. Yu, M. Batzill, J. He, U. Diebold, and J. Tang, Phys. Rev. B 73, 134412 (2006).

3. Y-w. Jun, J-s. Choi, and J. Cheon, Chem. Commun. 12, 1203 (2007),

4. M. L. Peterson, A. F. White, G. E. Brown, Jr., and G. A. Parks, Environ. Sci. Technol. 31, 1573 (1997).

5. B. A. Manning, J. R. Kiser, H. Kwon, and S. R. Kanel, Environ. Sci. Technol. 41, 586 (2007).

6. T. Kendelewicz, P. Liu. C. S. Doyle, G. E. Brown, Jr., E. J. Nelson, and S. A. Chambers, Surf. Sci. 424, 219 (1999).

7. V. S. Coker, N. D. Telling, G. van der Laan, R. A. D. Pattrick, C. I. Pearce, E. Arenholz, F. Tuna, R. E. P. Winpenny, and J. R. Lloyd, ACS Nano 3, 1922 (2009).

8. R. S. Cutting, V. S. Coker, N. D. Telling, R. L. Kimber, C. I. Pearce, B. Ellis, R. Lawson, G. van der Laan, R. A. D. Pattrick, D. J. Vaughan, E. Arenholz, and J. R. Lloyd, Environ. Sci. Technol., submitted (2009).

9. S. J. Brotton, R. Shapiro, G. van der Laan, J. Guo, P. A. Glans, and J. M. Ajello, J. Geophys. Res. 112, G03004 (2007).

10. G. van der Laan and I. W. Kirkman, J. Phys.: Condens. Matter 4, 4189 (1992).

11. M. Mizumaki, A. Agui, Y. Saitoh, M. Nakazawa, T. Matsushita, and A. Kotani, Surf. Rev. Lett. 9, 849 (2002).

12. R. A. D. Pattrick, G. van der Laan, C. M. B. Henderson, P. Kuiper, E. Dudzik, and D. J. Vaughan, Eur. J. Mineral. 14, 1095 (2002). 
Figure captions

FIG. 1: XA spectra of the $\mathrm{Cr}$ and $\mathrm{Fe} L_{2,3}$ edges for magnetite nanoparticles treated with solutions of varying $\mathrm{Cr}(\mathrm{VI})$ concentration.

FIG. 2: (color online) (a) $\mathrm{Cr} L_{2,3} \mathrm{XA}$ spectra for nanoparticles treated with $5 \mathrm{mM}$ solution (top) compared to standards obtained from ref. 6. (b) XMCD spectra for nanoparticles treated with solutions of varying $\mathrm{Cr}(\mathrm{VI})$ concentration (top). The XMCD spectra were obtained after first normalising the (background subtracted) XA spectra to unity at the $\mathrm{L}_{3}$ maximum. The lower curve is a charge transfer multiplet calculation reproduced from ref. 11 .

FIG. 3: (color online) Fe $L_{3}$ XMCD measured from untreated magnetite nanoparticles (dashed blue curve) compared to (a) nanoparticles treated with $5 \mathrm{mM} \mathrm{Cr}(\mathrm{VI})$ solution and (b) Co doped magnetite nanoparticles (red solid curves). The data in (b) is taken from Ref. 7.

FIG. 4: (color online) (a) Fe $L_{2,3}$ XMCD spectra measured from untreated and $\mathrm{Cr}(\mathrm{VI})$ reacted magnetite nanoparticles (black circles) together with corresponding best-fit curves (red drawn curves). The corresponding scaled cation site components used for the best-fit curves are shown in (b) and (c).

FIG. 5: (color online) (a) Change in $\mathrm{Fe}^{3+}$ and $\mathrm{Fe}^{2+}$ octahedral (Oh) site occupancy relative to the untreated sample, determined from fitting the XMCD spectra. (b) Net change in $\mathrm{Fe}^{2+} \mathrm{Oh}$ occupation (corrected for oxidation $\mathrm{Fe}^{2+} \rightarrow \mathrm{Fe}^{3+}$ ), obtained by the sum of the curves in (a). Lines are guides to the eye. 


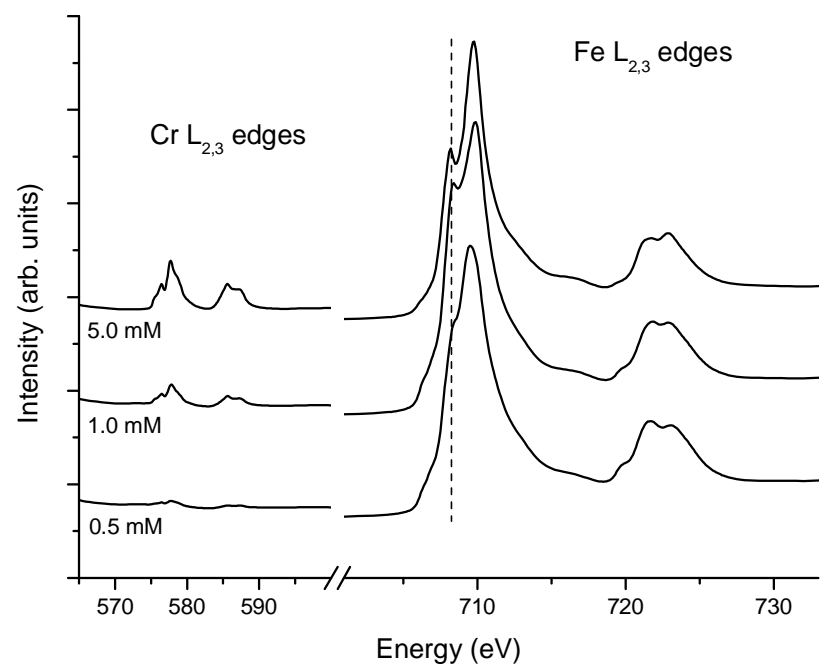

Fig. 1

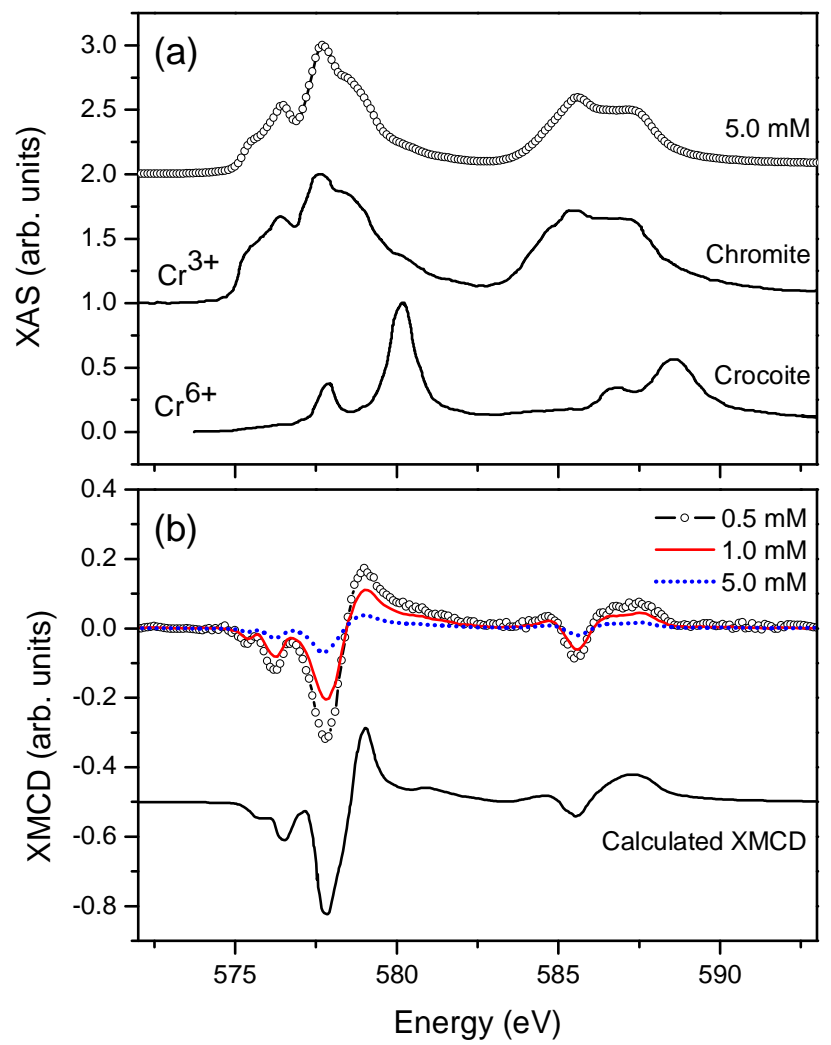

Fig. 2 


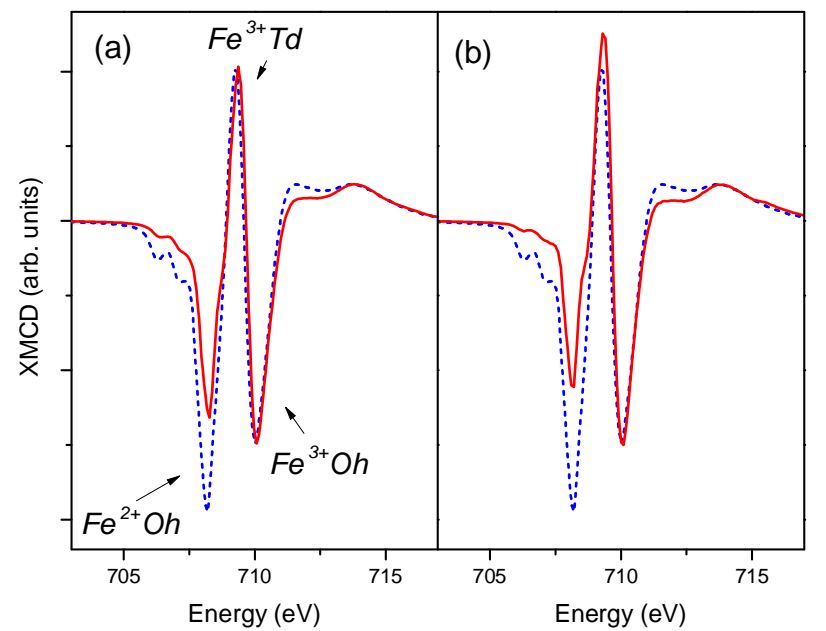

Fig. 3
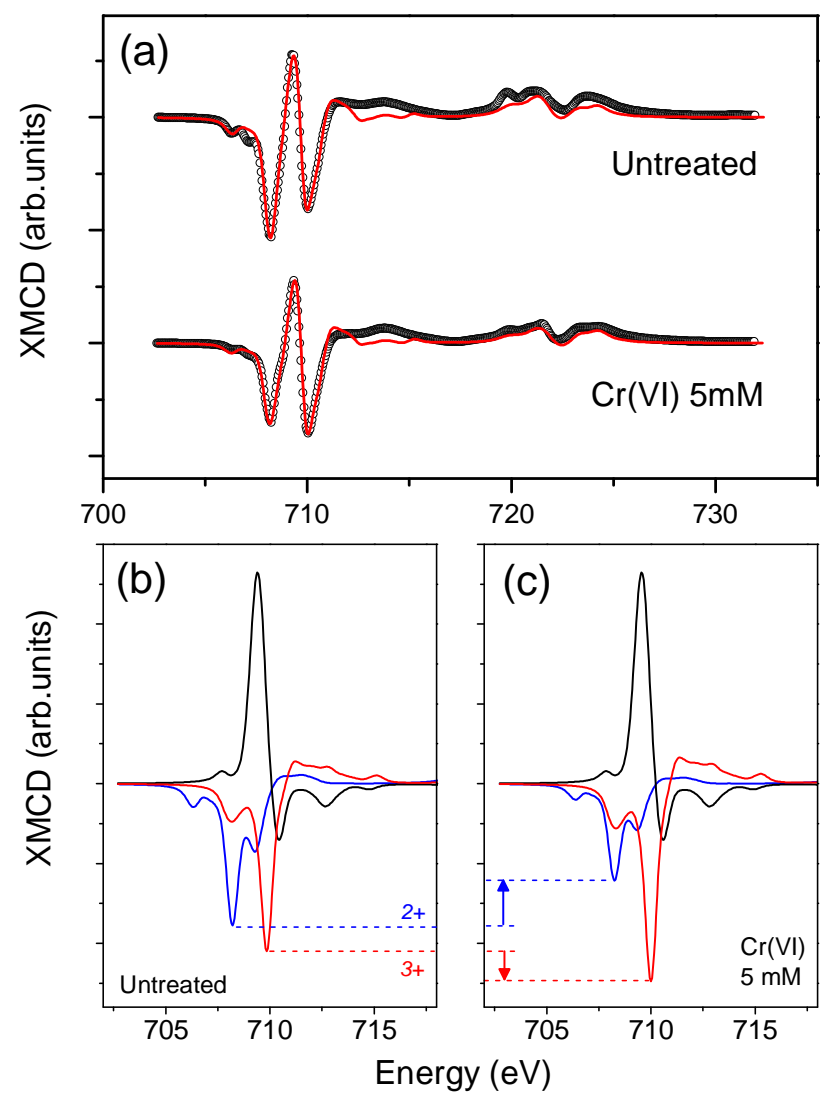

Fig. 4 


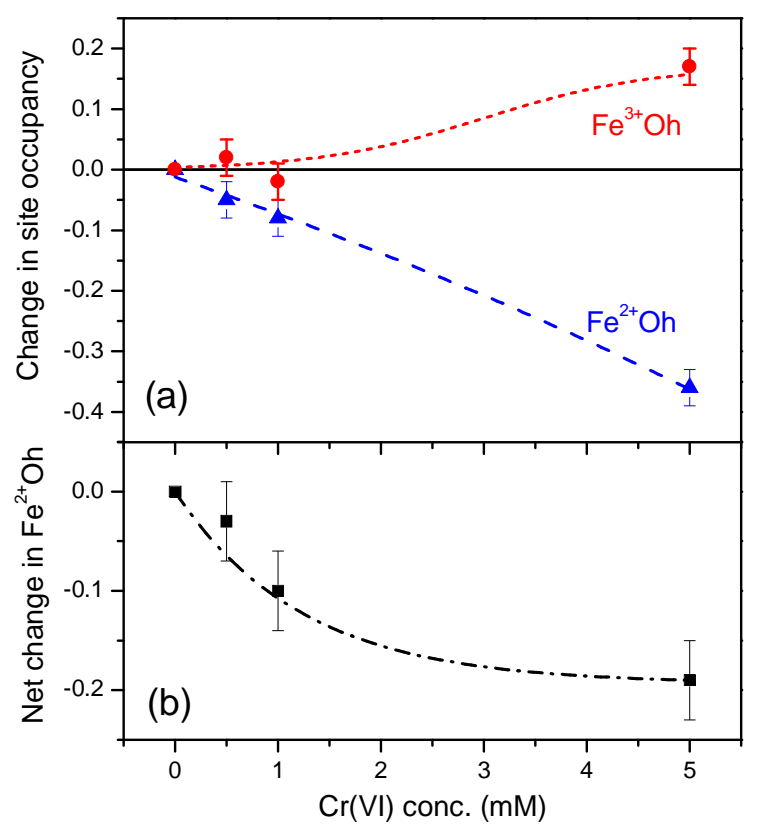

Fig. 5 